\title{
Crowd sourced smart EV charging station network using ML
}

\author{
Spuritha $\mathrm{M}^{1}$, Harshitha Damineni ${ }^{2}$, Shreemayi Sonti ${ }^{2}$, Veshala Lokesh Kumar ${ }^{2}$, Siri Chandana Veeramalla ${ }^{1}$, \\ Ramprasad Kokkula ${ }^{2}$, Dr. D.Indira ${ }^{3}$ \\ ${ }^{1}$ G.Narayanamma Institute of Technology and Science, Dept. of Information Technology, Hyderabad, Telangana, India \\ ${ }^{2}$ Gokaraju Rangaraju Institute of Engineering \& Technology, Department of Electronics and Communications Engineering, Bachupally, \\ Hyderabad,India \\ ${ }^{3}$ Gokaraju Rangaraju Institute of Engineering \& Technology, Department of Humanities and Sciences , Bachupally, Hyderabad,India
}

\begin{abstract}
Electric vehicle owners face the problem of having limited charging station options. Individual charging stations near households can act as a viable solution to solve this problem. A forecasting model which can effectively predict the power consumption of a charging station will help charging station owners get a clear view of how much energy to produce. With this intent, this paper proposes an Internet of Things (IoT) based charging station network that acts as a platform to provide charging to electric vehicles and a model based on ARIMA whose learners are fitted to the charging station subsets with optimum parameters to increase the overall performance of sales prediction. The proposed model predicted power consumption for 7 charging stations, with average MAPE, RMSE and $\mathrm{R}^{2}$ values of $12.88 \%, 5.67$, and 0.79 respectively.
\end{abstract}

\section{Introduction}

With the rising adoption of electric vehicles, the automobile industry is undergoing rapid change. This necessitates the construction of a massive infrastructure of electric charging stations. According to a Business Standard research published in 2021, India will require 4,00,000 charging stations by 2026 to accommodate an anticipated 4 million electric vehicles [1]. The installation of an electric charging station is an expensive process, with an average cost of $25,00,000$ INR. The majority of the costs are related to obtaining land cover and maintaining a steady supply of electricity. Setting up charging stations in residential areas could be a viable solution for providing cheaper land [2]. Using renewable energy to generate power can result in lower electricity costs [3].

Connecting charging stations to the internet and establishing a smart charging station allows consumers to schedule a charging station through a website or mobile app [4]. While smart charging stations have their advantages in this situation, automating the charging process and estimating the number of consumers on any given day is a hassle [5]. To address these issues, an IoT-based interconnected electric charging station is proposed, consisting of a network of automated charging stations that can be booked online. To effectively manage the charging station's energy source, an algorithm based on the ARIMA model is utilized to estimate daily sales for the charging station [6][7].

\section{Proposed Method}

A network of IoT-based charging stations is being established, and these charging stations can be booked through a website. Thecharging stations are connected to the cloud. A current sensor and a relay module are used in charging stations. On a regular basis, the data acquired from the sensors is uploaded to the cloud.

All the information about the clients who booked charging stations is saved. Customers will be given an authentication token that they can use to gain access to the charging station. The charging process is regulated by the current sensor based on the charging percentage selected by the customer on the website. The relay module is used to turn on and off the charging station's power supply. It is updated in the cloud, after the charging process is completed.

If a customer wants to book a charging station, they should go to the website and choose their location. The website dynamically displays the available charging stations based on the user's location .The charging station can be used by the customer after arriving at the designated charging station location and entering an authentication token provided to the customer after booking confirmation. The charging station list in a location is updated hourly on the website based on its availability, as shown in Fig. 1. 


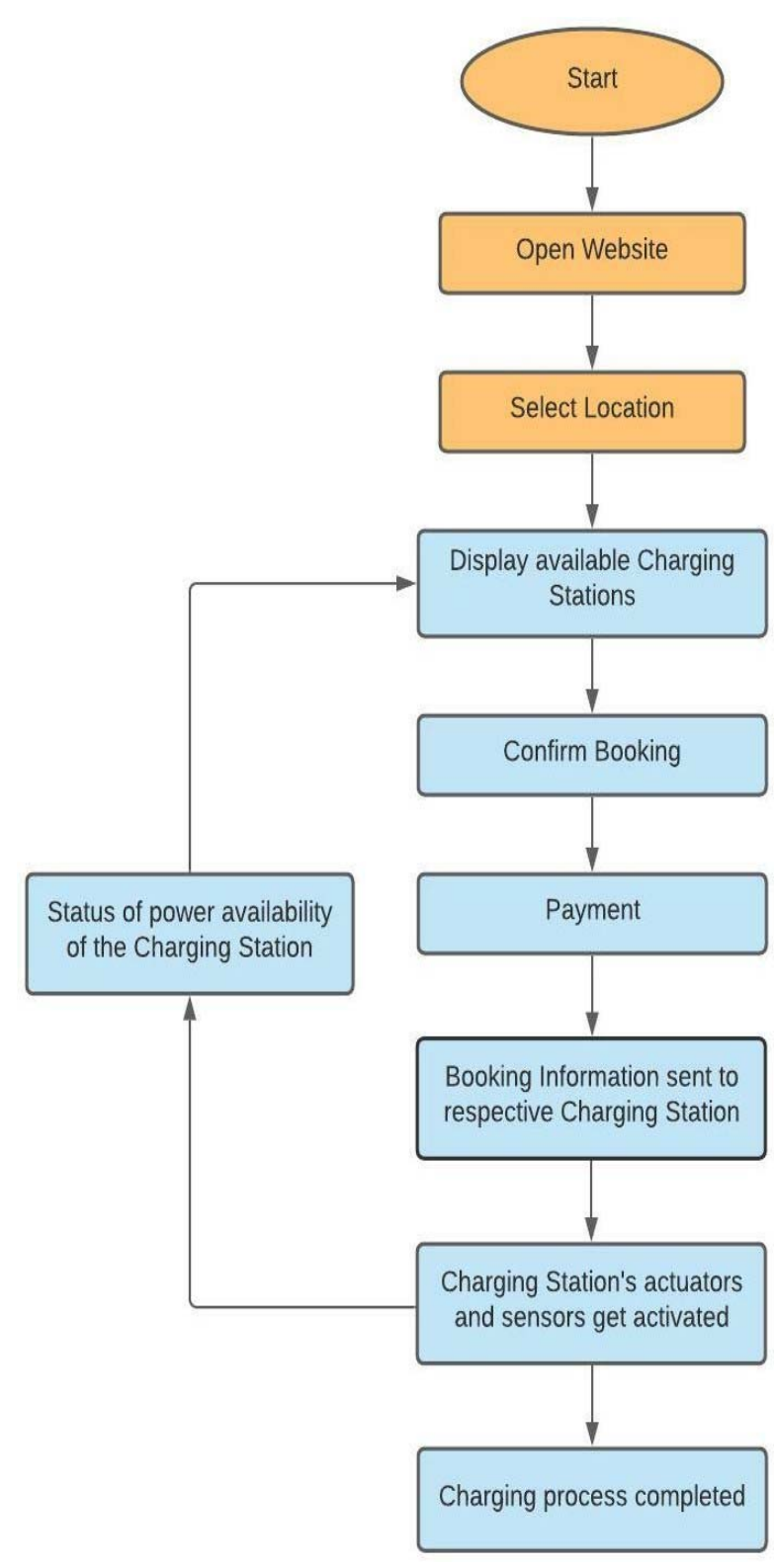

Fig. 1. Implementation of proposed system

The booking information is transferred to the cloud, and the authentication token information is forwarded to the charging stations that have been specified. After that, the authentication token can be used to authorize the customers. The list of available stations is updated on a regular basis by updating the charging station status after each successful order. Charging stations as shown in Fig. 2 are the devices available to a customer in a desired location

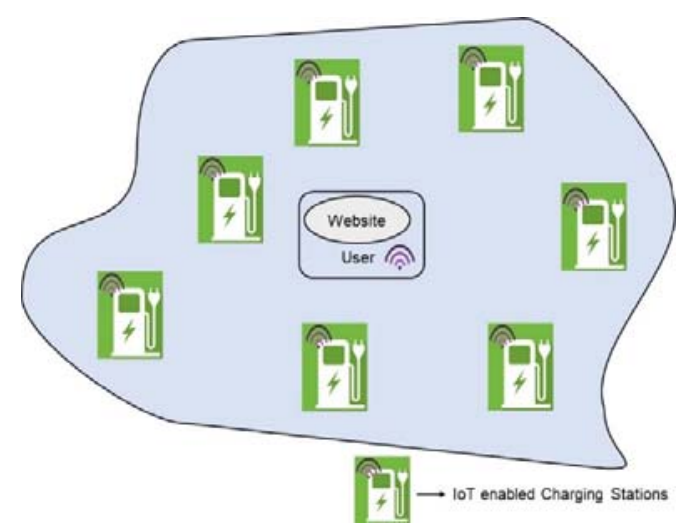

Fig. 2. Model of the proposed system

Data was collected from seven charging stations in an area forthe year 2020 to design a machine learning-based energy consumption forecasting model.

To obtain insight into product sales at various stores over time, the dataset is exposed to exploratory data analysis (EDA), and several charts are created. After that, the dataset is subjected to feature engineering, which extracts important sub-features from the original features. Feature engineering helps develop a stronger machine learning model byexpanding the dimensionality of the dataset. The dataset is divided into training and testing partitions. After the splitting method, time series forecasting is utilized to build the prediction model.

\section{Implementation}

A system of six charging stations is implemented where each charging station gets its input from the cloud that stores the order information received from the website. And performs physical action to charge the customer's vehicle to the desired charging percentage. The implementation of the proposed system is explained through the following subsections.

\subsection{Website}

A website is designed as shown in Fig. 3 which actsas a marketplace to book charging stations [8]. The following are the steps involved in developing the user interface web application for the proposed system:

Step 1: The website is designed using React JS as the frontend framework and Express JS as the backend framework.

Step 2: Product details are rendered from Firebase based on the vending machine selected by the user.

Step 3: Authentication of the users and extraction of their user history is also done using Firebase.

Step 4: A State container is created using redux for processing order information.

Step 5: Payment interface is created using RazorPay.

Step 6: Verification of users and intimating the user about the package delivery status is done using Twilio.

Step 7: The authentication tokens of the booking are stored in Firebase cloud 


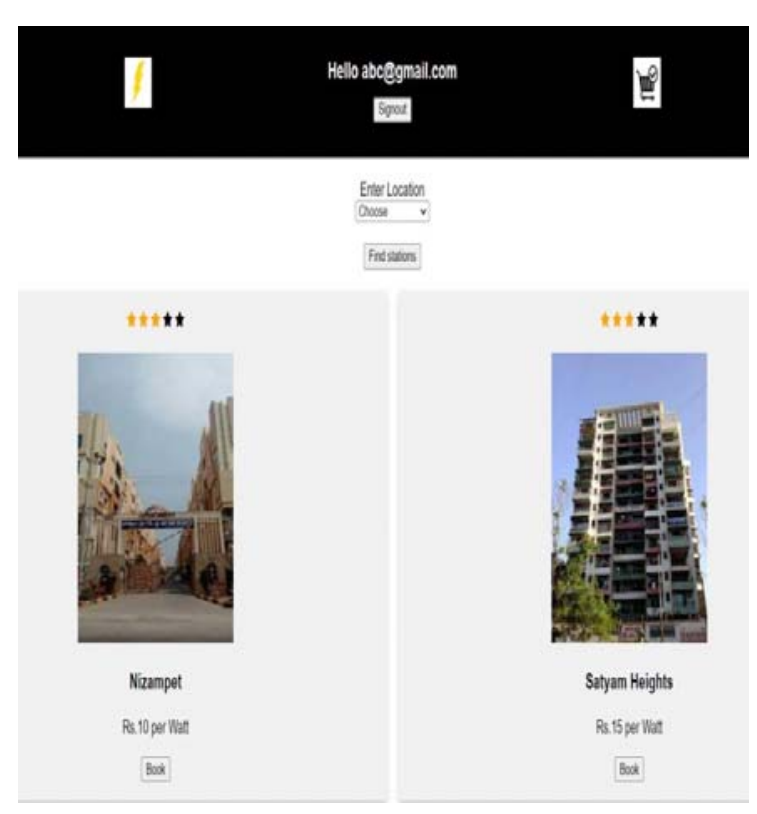

Fig. 3. Designed website user interface

\subsection{Charging Station}

The charging stations in the proposed system act as a power vending machine [9]. These charging stations consist of a constant power supply, acurrent flow sensor and a relay module. As mentioned before, the sensor is used to regulate the amount of charge that is flown out from the charging station. Whenever the charging station gets a booking authentication token from the cloud via ESP8266 module, the relay module allows the current flow till the current sensor notifies the microcontroller that thedesired charging is reached. After the completion of the task a message is sent to the cloud regarding the task accomplishment.

\subsection{Machine Learning}

The data set, which was collected from retail shops, includes the date, timestamp, location and meter value [10][11]. The proposed system's implementation is shown in Fig and described in the following subsections.

\subsubsection{Load data}

The data set is saved in a CSV file format. For the year 2020, the data set has three columns and 1,50,000 rows. As shown in Table I, the data set includes columns for station id and meter value for thevarious dates.

Table.1: Sample records of the dataset

\begin{tabular}{|c|c|c|c|}
\hline S. No. & Date & Station Id & $\begin{array}{l}\text { Meter } \\
\text { Values } \\
(\mathrm{kW})\end{array}$ \\
\hline 1 & 2020-01-01 & 3 & 1013 \\
\hline & 2020-01-02 & 3 & 1007 \\
\hline 3 & 2020-01-03 & 3 & 1054 \\
\hline 4 & 2020-01-04 & 3 & 1097 \\
\hline 5 & $2020-01-05$ & 3 & 1029 \\
\hline 6 & 2020-01-06 & 3 & 1059 \\
\hline 7 & 2020-01-07 & 3 & 1077 \\
\hline 8 & 2020-01-08 & 3 & 1063 \\
\hline 9 & 2020-01-09 & 3 & 1099 \\
\hline 10 & 2020-01-10 & 3 & 1010 \\
\hline
\end{tabular}

\subsubsection{Preprocessing and Visualizing dataset}

The seasonality of the input dataset is found using time series decomposition. This technique is used to decompose the time series data into trend, seasonality and residual components [12][13].

\subsubsection{Trend}

The Trend indicates the progression of sales over the year. 


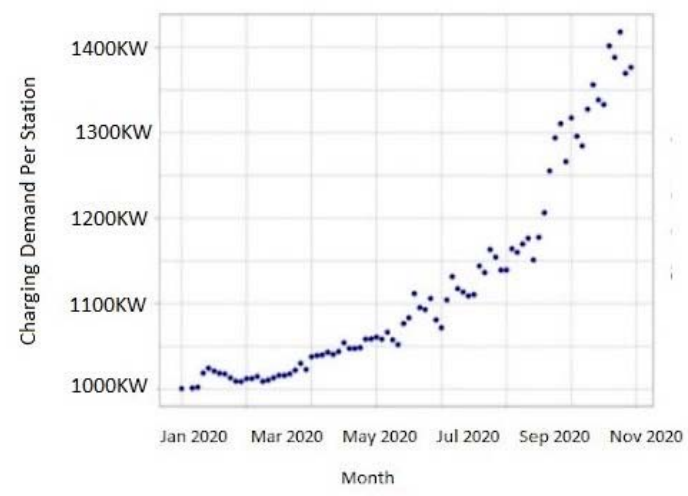

Fig. 4. Increasing trend of charging demand

\subsubsection{Residual}

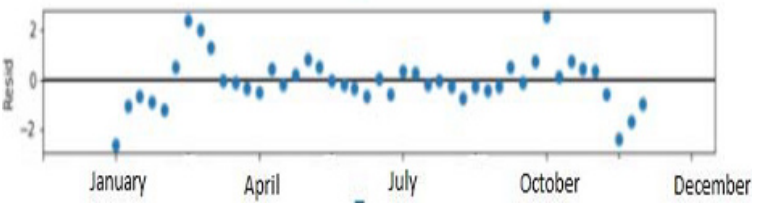

Fig. 5. Random noise in the data

\subsubsection{Stationarising Dataset}

The statistical features of a dataset, such as mean, variance, autocorrelation, and others, are checked for consistency across time to assess its stationarity. DickeyFuller test can be used to determine if the series is stationary or not.

We see that the $\mathrm{p}$-value is greater than 0.05 . To get a stationary series, we need to eliminate the trend and seasonality from the series [14]. Computing the differences between consecutive observations is the first difference that makes the non stationary series stationary. If after taking the first difference also doesn't result in $\mathrm{p}$ being less than 0.05 (Non Stationary), differencing is performed again until the sequence becomes stationary.

\subsubsection{Parameter Determination}

We must first find the best parameters for our forecasting model before proceeding. We'll need ACF and PACFgraphs to get those ideal settings [15].

\subsubsection{Autocorrelation Function(ACF)}

The connection between time series observations and prior time steps, known as lags, may be calculated. The ACF plot shows the autocorrelations which measure the relationship between $\mathrm{y}(\mathrm{t})$ and $\mathrm{y}(\mathrm{t}-\mathrm{k})$ for different values of $\mathrm{k}$. ACF is exponentially decaying or sinusoidal. There is a significant spike at lag $\mathrm{p}$ in the PACF, but none beyond lag $\mathrm{p}$.

\subsubsection{Partial Autocorrelation Function(PACF)}

PACF shows the relationship between $y(t)$ and $y(t-k)$ after removing the effects of lags $1,2,3, \ldots, \mathrm{k}-11,2,3, \ldots, \mathrm{k}-1$. PACF is exponentially decaying or sinusoidal. There is a significant spike atlag $\mathrm{q}$ in the ACF, but none beyond lag q.

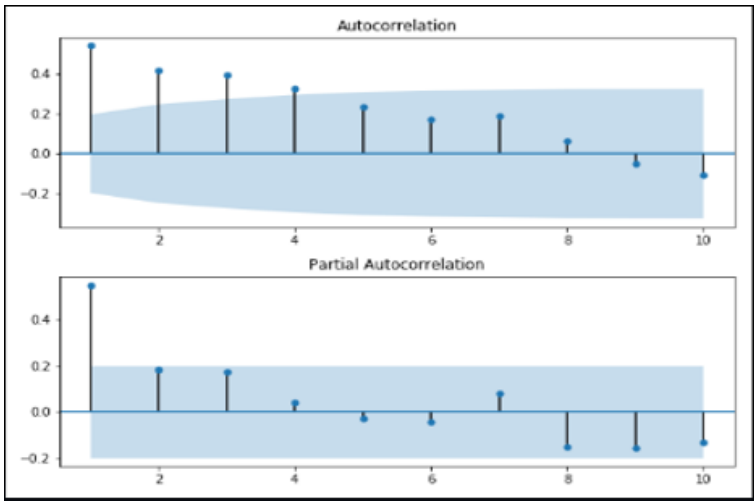

Fig. 6. Auto correlation and partial auto correlation plots

\subsubsection{Predictions}

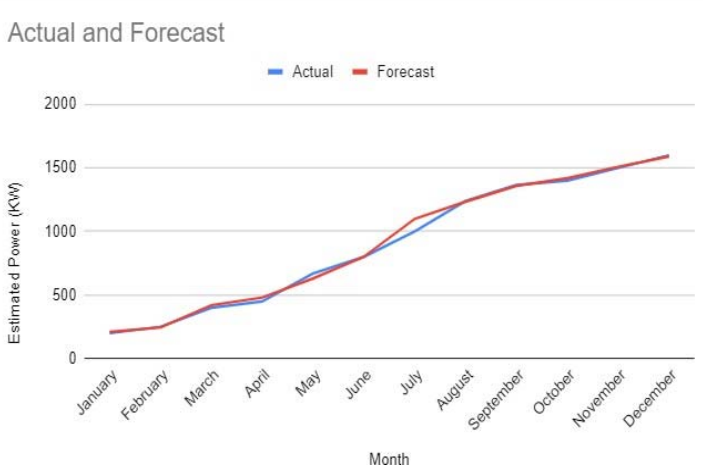

Fig. 7. Comparison between actual and total predicted power

The last three months of the dataset are utilized to evaluate the algorithm and forecast power consumption of a charging station per day for the same time period. The actual and predicted power consumption in the test set and the errors are shown in the figure 7 . The total power consumption from the test set for the charging station 2 was 10876, whereas the predicted power consumption is 11001 .

\section{$4 \quad$ Results and Discussion}

The metrics used to assess the proposed system's accuracy are Mean Absolute Percentage Error (MAPE), Root Mean Square Error (RMSE), and R2 score.

Mean Absolute Percentage Error: 
MAPE is the average of the absolute percentage errors of forecasts as in (1). The average MAPE of the proposed model is $11.98 \%$.

$$
\begin{aligned}
M= & \frac{1}{n} \sum_{t=1}^{n} \quad\left|\frac{A_{t}-F_{t}}{A_{t}}\right| * 100 \\
& \mathrm{M} \text { - MAPE } \\
& \mathrm{n} \text { - number of fitted points } \\
& A_{t} \text { - actual value } \\
& F_{t} \text { - forecast value }
\end{aligned}
$$

Root Mean Square Error:

It's the root of the mean of squared differences between actual and predicted observations as in (2). The proposed model has an average RMSE value of 63

$$
\begin{aligned}
\text { RMSE }= & \sqrt{\frac{1}{n} \sum_{j=1}^{n}\left(y_{j}-\hat{y}_{j}\right)^{2}} \\
& y_{j} \text { - actual value } \\
& \hat{y}_{j} \text { - predicted value } \\
& \mathrm{n} \text { - number of observations }
\end{aligned}
$$

$\mathrm{R}^{2}$ score:

For regression models, the $\mathrm{R} 2$ score is a measure of goodness-of-fit. R2 is calculated by finding the variation of the difference of sum of squares and total sum as in (3) The proposed model's average R2 score is 0.76 , indicating that it is better at fitting the data.

$$
\begin{gathered}
R^{2}=1-\frac{S S_{\text {res }}}{S S_{\text {tot }}}(3) \\
S S_{\text {res }} \text { - Sum of squares of the residual errors } \\
S S_{\text {tot }} \text { - Total sum of the errors }
\end{gathered}
$$

The last six months of the dataset are utilized to evaluate 019 . the algorithm and forecast power consumption per day for thEmbedded Drive Control (ICPEDC), (2019), pp. 342same time period. Fig. 5 indicates the depiction of power usage from the train, test sets, and predicted power usage.

Table 2: Metrics used for evaluation of the proposed model

\begin{tabular}{|l|l|l|l|}
\hline & AverageMAPE & AverageRMSE & Average R`2 \\
\hline ARIMA & $12.88 \%$ & 5.67 & 0.79 \\
\hline
\end{tabular}

It can be inferred from Table II, that the average MAPE, RMSE and $\mathrm{R}^{2}$ values across all products and store models ar $12.88 \%, 5.67$, and 0.79 .

\section{$5 \quad$ Conclusion}

The issue of a lack of charging stations can be addressed by12. Prasad, K.S., Gupta, A.K, Singh, Y. Singh, S.K. $A$ establishing an integrated decentralized network of chargingModified Mechanical Threshold Stress Constitutive

stations, and a machine learning forecasting algorithm can as Model for Austenitic Stainless Steels (2016) Journal of sist in predicting energy consumption on a given day.

\section{References}

1. Mishra S, Verma S, Chowdhury S, Gaur A, Mohapatra S, Dwivedi G, Verma P. A Comprehensive
Review on Developments in Electric Vehicle Charging Station Infrastructure and Present Scenario of India. Sustainability. (2021);13(4):2396.

2. D. Sbordone, I. Bertini, B. Di Pietra, M.C. Falvo, A. Genovese, L. Martirano, EV fast charging stations and energy storage technologies: A real implementation in the smart micro grid paradigm,Electric Power Systems Research,Volume 120,(2015).

3. Ibrahim Dincer, Renewable energy and sustainable development: a crucial review, Renewable and Sustainable Energy Reviews, Volume 4, Issue 2,(2000).

4. Fang He, Yafeng Yin, Jing Zhou,Deploying public charging stations for electric vehicles on urban road networks, Transportation Research Part C: Emerging Technologies,Volume 60,(2015).

5. Hua Qin and Wensheng Zhang. Charging scheduling with minimal waiting in a network of electric vehicles and charging stations. In Proceedings of the Eighth ACM international workshop on Vehicular internetworking (VANET '11). Association for Computing Machinery, New York, NY, USA, 51-60.( 2011)

6. M. Majidpour, C. Qiu, P. Chu, R. Gadh and H. R. Pota, "Modified pattern sequence-based forecasting for electric vehicle charging stations," 2014 IEEE International Conference on Smart Grid Communications (SmartGridComm), (2014), pp. 710715 .

7. Hui Zhou, Qingzhu Chen and Rong Cong, "Forecasting model of charging load for electric vehicle based on mean test," 2014 IEEE Conference and Expo Transportation Electrification Asia-Pacific (ITEC AsiaPacific), (2014), pp.1-5.

8. R. George, S. Vaidyanathan and K. Deepa, "Ev Charging Station Locator With Slot Booking System," 348.

9. S. Bai, D. Yu and S. Lukic, "Optimum design of an EV/PHEV charging station with DC bus and storage system," 2010 IEEE Energy Conversion Congress and Exposition, 2010, pp. 1178-1184.(2010)

10. H. Chung, W. Li, C. Yuen, C. Wen and N. Crespi, "Electric Vehicle Charge Scheduling Mechanism to Maximize Cost Efficiency and User Convenience," in IEEE Transactions on Smart Grid, vol. 10, no. 3, pp. 3020-3030, May 2019.

11. Nirmala Devi, G., Chitra, S., Selvasekarapandian, S., Premalatha, M., Monisha, S., Saranya, J. Synthesis and characterization of dextrin-based polymer electrolytes for potential applications in energy storage devices (2017) Ionics, 23 (12), pp. 3377-3388.(2017) Materials Engineering and Performance, 25 (12), pp. 5411-5423.(2016)

13. Pandey, U., Purohit, R., Agarwal, P., Kumar Singh, S. Study of Fabrication, Testing and Characterization of Al/TiC Metal Matrix Composites through different Processing Techniques (2018) Materials Today: Proceedings, 5 (2), pp. 4106-4117.(2018) 
14. Patel, S., Rana, R.S., Singh, S.K. Study on mechanical properties of environment friendly Aluminium E-waste Composite with Fly ash and E-glass fiber (2017) Materials Today: Proceedings, 4 (2), pp. 3441-3450.(2017)
15. Dhanalaxmi, B., Apparao Naidu, G., Anuradha, K. Adaptive PSO based association rule mining technique for software defect classification using ANN (2015) Procedia Computer Science, 46, pp. 432-442.(2015) 